\title{
INFLUÊNCIA DA DENSIDADE DO SOLO NA ESTIMATIVA DA UMIDADE EM UM NITOSSOLO VERMELHO DISTROFÉRRICO, POR MEIO DA TÉCNICA DE TDR(1)
}

\author{
Antônio Carlos Andrade Gonçalves ${ }^{(2)}$, Marcos Antonio Trintinalha ${ }^{(3)}$, \\ Cássio Antônio Tormena ${ }^{(2)} \&$ Marcos Vinicius Folegatti ${ }^{(4)}$
}

\begin{abstract}
RESUMO
Entre as técnicas para avaliação da umidade do solo, destaca-se a que utiliza a Reflectometria no Domínio do Tempo (TDR), a qual consiste em um método indireto, não destrutivo, rápido, exato e pouco dependente dos fatores ambientais. Porém, trabalhos desenvolvidos após as suas aplicações sugerem a influência de fatores ligados às características mineralógicas e estruturais dos solos, o que torna necessária a calibração local para o adequado funcionamento da TDR. O objetivo deste trabalho foi estudar a influência da densidade do solo (Ds) na medida da umidade volumétrica do solo $(\theta)$, utilizando-se a técnica de TDR, em um Nitossolo Vermelho distroférrico. Um modelo que expressa a relação entre a constante dielétrica do solo (Ka) e $\theta$ foi ajustado por meio de regressão polinomial múltipla, com a incorporação da Ds para a estimativa dos seus coeficientes. Os resultados permitiram concluir que, nesse solo, o modelo de calibração deve levar em conta o efeito da Ds, sem o que a qualidade das estimativas de umidade, a partir das medidas de constante dielétrica, fica comprometida. Os resultados evidenciaram também que a sensibilidade da TDR à variação da umidade do solo é elevada, tornando possível que a técnica seja utilizada com exatidão em ações que envolvam o monitoramento da variação da umidade do solo. Os resultados indicaram a necessidade de mais estudos sobre a utilização da TDR em solos tropicais.
\end{abstract}

Termos de indexação: reflectometria, irrigação, água no solo, estrutura do solo, instrumentação.

\footnotetext{
(1) Recebido para publicação em 11 de maio de 2010 e aprovado em 15 de junho de 2011.

(2) Professor do Departamento de Agronomia da Universidade Estadual de Maringá - UEM. Av. Colombo 5790, CEP $87020-900$ Maringá (PR). E-mail:acagoncalves@uem.br

(3) Engenheiro-Agrônomo e D.S. em Solos e Nutrição de Plantas, UEM. E-mail: dnn@cocari.com.br

(4) Professor Titular do Departamento de Engenharia Rural, Escola Superior de Agricultura "Luiz de Queiroz" - ESALQ/USP. Caixa Postal 9, Av. Pádua Dias 11, CEP 13418-900 Piracicaba (SP). E-mail: mvfolega@esalq.usp.br
} 


\title{
SUMMARY: INFLUENCE OF SOIL BULK DENSITY IN A DYSTROFERRIC RED ULTISOL MOISTURE ESTIMATED BY TIME DOMAIN REFLECTOMETRY
}

\begin{abstract}
Among the techniques for soil moisture evaluation, the use of Time Domain Reflectometry (TDR) stands out, which consists of an indirect, non-destructive, fast and accurate method less dependent on environmental factors. However, works carried out after its applications suggest the influence of factors related to soils structural and mineralogical characteristics, which makes necessary the site calibration for the TDR proper functioning. The aim of this work was to study the influence of bulk density (Ds) on the volumetric soil water content $(\theta)$, using the TDR technique in a Dystroferric Red Ultisol. A model that expresses the relationship between the soil dielectric constant (Ka) and $\theta$ was adjusted by multiple polynomial regression, with the incorporation of Ds for the estimation of its coefficients. The results showed that in this soil, the calibration model should take into account the effect of Ds, without which the quality of the moisture estimates from the dielectric constant measurements is compromised. The results also highlighted the high sensitivity of TDR to the variation of soil moisture, making it possible the accurate use of this technique in actions involving the monitoring of the variation in soil moisture. The results indicated the need for further studies on the use of TDR in tropical soils.
\end{abstract}

Index terms: reflectometry, irrigation, soil water, soil structure, instrumentation.

\section{INTRODUÇÃO}

A umidade do solo é uma variável fundamental no estudo e modelagem de diferentes processos agronômicos, hidrológicos e ambientais (Tsegaye et al., 2004). As variabilidades temporal e espacial da umidade volumétrica do solo $(\theta)$ relacionam-se com a maioria desses processos, de modo que sua quantificação requer técnicas que permitam medidas rápidas, exatas, passíveis de automação e de repetição no mesmo local, com o mínimo de perturbação do solo.

De acordo com Topp \& Reynolds (1998), a técnica de TDR revolucionou o processo de determinação da umidade do solo. Outros métodos, tradicionalmente empregados, apresentam limitações. O método termogravimétrico é direto e utilizado como padrão para determinar $\theta$. Porém, esse é um método trabalhoso, de ação lenta e destrutiva. Métodos indiretos são disponíveis, como a técnica de termalização de nêutrons (sonda de nêutrons) que, apesar das vantagens, apresenta risco potencial da radioatividade para a saúde humana e para o ambiente, além de tornar necessária a sua calibração local. Nesse contexto, destaca-se a técnica de TimeDomain Reflectometry (TDR) ou reflectometria no domínio do tempo, que mede a constante dielétrica do solo (Ka), cujo valor é relacionado com os valores de $\theta$. A TDR foi empregada em meios porosos desde que Topp et al. (1980) propuseram uma relação empírica entre a constante dielétrica e $\theta$. Kameyama \& Miyamoto (2008) apresentam essa relação por meio da equação $\mathrm{Ka}=3,03+9,3 \theta+146 \theta^{2}-76,7 \theta^{3}$, destacando que esses coeficientes numéricos poderiam ser assumidos como universais.

A técnica da TDR foi utilizada pela primeira vez em Ciência do Solo por Topp et al. (1980). Consiste na determinação da constante dielétrica aparente do meio (Ka) a partir da medida do tempo de viagem de um pulso eletromagnético em uma sonda metálica de certo comprimento, cravada no solo, na profundidade desejada (Fellner-Feldegg, 1969). Os princípios e as aplicações potenciais da TDR são revisados por Noborio (2001) e Jones et al. (2002). A utilização da TDR tem despertado muita atenção, pois é uma técnica não destrutiva, de manuseio fácil e elevada exatidão (Topp et al., 1980; Topp \& Davis, 1982; Topp et al., 1984; Timlin \& Pachepsky, 1996; Wang et al., 1998; Walker et al., 2004) e sem os riscos de radiação (Robinson et al., 1994).

Uma das características mais atrativas da técnica da TDR é o seu potencial de uso para monitorar, contínua e simultaneamente, a umidade do solo em vários locais (Baker \& Allmaras, 1990). Além disso, Zegelin et al. (1989) indicam que a técnica apresenta a vantagem da rapidez, uma vez que realiza amostragens em pequenos intervalos de tempo. Alaoui \& Helbling (2006) destacam a vantagem da TDR pelo fato de ela possibilitar o monitoramento de grande volume de solo, levando em consideração o domínio tanto da microporosidade quanto da macroporosidade. Schneider \& Fratta (2009) destacam a influência da porosidade do material próximo à sonda sobre a medida da constante dielétrica, especialmente para valores de umidade próximos da saturação.

A utilização do modelo apresentado por Topp et al. (1980) foi consolidada a partir de estudos que apontaram o fato de que o valor de Ka dependia fortemente da umidade do solo, sendo essa relação mais expressiva que o efeito de outras variáveis relacionadas com textura, temperatura, estrutura e salinidade do solo (Hipp, 1974; Hoekstra \& Delaney, 1974; Davis \& Annan, 1977; Topp et al., 1980; Keng \& Topp, 1983). 
Esses trabalhos indicavam que o modelo de Topp et al. (1980) poderia ser utilizado como uma única função de calibração entre $\theta$ e Ka. No entanto, trabalhos posteriores evidenciaram que o modelo proposto por Topp et al. (1980) tinha validade restrita para alguns solos minerais, sugerindo a necessidade de calibração local do equipamento (Heathman et al., 2003; Jackson, 2004). De acordo com Tsegaye et al. (2004), a utilização de sensores para a estimativa da umidade volumétrica do solo demanda calibração para o local específico, em função da variabilidade do solo e dos efeitos relacionados à constituição do material de solo. Por exemplo, em solos orgânicos e em solos argilosos, vários estudos mostram a necessidade de estabelecer funções específicas entre Ka e $\theta$ (Ponizovsky et al., 1999; Roth et al., 1992). Tommaselli \& Bacchi (2001) obtiveram uma curva de calibração para cinco solos tropicais, concluindo que ela não poderia ser considerada universal e que o modelo de Topp et al. (1980) não seria aplicável aos solos estudados. Aspectos específicos da configuração do equipamento utilizado para medir as características dielétricas do meio foram discutidos por Pettinelli et al. (2002). Kameyama \& Miyamoto (2008) sinalizam com a perspectiva de que a microestrutura do solo influencia nos valores obtidos para Ka, o que deve ser considerado na utilização desse método para medir a umidade do solo.

Embora a medida de Ka realizada com a TDR seja fortemente relacionada com o valor de $\theta$, ela é afetada por inúmeros fatores, como a salinidade (Bonnell et al., 1991), a presença e concentração de minerais de $\mathrm{Fe}$, particularmente minerais magnéticos (Roth et al., 1992; Robinson et al., 1994), a textura (Roth et al., 1992; Bridge et al., 1996; Ponizovsky et al., 1999), a área superficial específica (ASE) dos solos (Jones \& Or, 2003) e a variação da temperatura do solo durante a leitura. Em solos atípicos como os de origem vulcânica (ândicos), com elevada presença de minerais amorfos e de baixa densidade do solo, têm-se constatado modificações no sinal da TDR, o que também demonstra o efeito particular da constituição do solo e a necessidade de modelos de calibração para essas condições (Todoroff \& Langellier, 1998). Jones \& Or (2003) consideram que a ASE dos constituintes do meio poroso desempenha papel fundamental nas medidas de TDR, o que pode explicar o desvio do modelo de Topp em relação aos solos com ASE muito diferentes.

As modificações na estrutura do solo expressas pelo aumento da densidade e redução da porosidade podem ter potencial de alteração da constante dielétrica do solo para determinado valor de $\theta$, o que pode implicar mudanças na curva de calibração da TDR. No trabalho de Roth et al. (1992), comparando diferentes solos, há indicação de que a densidade do solo desempenha papel importante na relação Ka versus $\theta$. No entanto, os resultados de Rothe et al. (1997) indicam que a compactação do solo por ocasião da instalação das sondas pode causar alterações substanciais nas estimativas de umidade pela TDR, principalmente em elevados valores de umidade. Em solos vulcânicos caracterizados pela reduzida densidade e elevados teores de matéria orgânica, Weitz et al. (1997) constataram que o modelo proposto por Topp subestimou $\theta$ em até $15 \%$. Malicki et al. (1996), utilizando diferentes materiais porosos, inclusive solo, demonstraram que a densidade, assim como a porosidade, influenciam na relação entre Ka e $\theta$ e que os erros das estimativas foram contornados quando os efeitos dessas variáveis foram considerados na função de calibração. Também Schaap et al. (1996) obtiveram melhores estimativas de $\theta$ em solos florestais, levando-se em conta o efeito da densidade na curva de calibração da TDR.

Os resultados apresentados por Jacobsen \& Schjonning (1993) apontam efeitos positivos de se considerar a densidade do solo na curva de calibração. Trabalhos posteriores, como o de Gardner et al. (1998), mostraram que as estimativas de $\theta$ por meio de um sensor de capacitância foram influenciadas pela densidade do solo. Em solos tropicais, trabalhos recentes como o de Coelho et al. (2006) evidenciaram que não é possível a generalização do modelo de calibração. Também Kaiser et al. (2010), trabalhando com solos brasileiros, identificaram a inadequação dos modelos gerais propostos, evidenciando a necessidade de modelos específicos para cada solo. Os referidos autores destacam que a relação entre umidade volumétrica e constante dielétrica varia fortemente com o conteúdo de argila e de óxidos de Fe.

De acordo com Robinson et al. (2005), a ausência de um modelo robusto e exato, com base física, promoveu o surgimento de grande número de modelos empíricos de calibração para a TDR. De acordo com Cerny (2009), mesmo após décadas de uso e desenvolvimento da técnica, ainda não há como ignorar o efeito das características do material sobre as medidas realizadas. Nesse sentido, ainda não foi possível o desenvolvimento de um modelo geral de calibração. Informações mais detalhadas a respeito dos efeitos da estrutura do solo nas relações entre Ka e $\theta$ são necessárias para avaliar a exatidão e aplicabilidade da TDR na medida de $\theta$. Dessa forma, os objetivos deste trabalho foram mensurar os efeitos da variação da densidade do solo na relação funcional entre Ka e $\theta$ e modelar esses efeitos, introduzindo o valor de Ds nos coeficientes do modelo ajustado aos dados experimentais, em um Nitossolo Vermelho distroférrico comumente encontrado nas regiões Norte e Noroeste do Estado do Paraná.

\section{MATERIAL E MÉTODOS}

O experimento foi conduzido na unidade de pesquisa em agricultura irrigada da Universidade Estadual de Maringá, localizada no município de Maringá, PR, com latitude de $23^{\circ} 25^{\prime}$ Sul e longitude de $51^{\circ} 57^{\prime}$ a Oeste de Greenwhich. A altitude média é de $540 \mathrm{~m}$ e o clima predominante, segundo a classificação de 
Köeppen, é do tipo Cfa, mesotérmico úmido com chuvas abundantes no verão e inverno seco, com precipitação pluvial média anual de $1.500 \mathrm{~mm}$. Nos meses de julho a agosto, concentram-se os menores índices de precipitação pluvial. A temperatura média anual é de $16,7^{\circ} \mathrm{C}$, em que a média das mínimas alcança $10,3{ }^{\circ} \mathrm{C}$ e a média das máximas atinge $33,6^{\circ} \mathrm{C}$. O solo da área experimental foi classificado, de acordo com a Embrapa (2006), como Nitossolo Vermelho distroférrico, com horizonte A moderado, classe textural muito argilosa, localizado em relevo suaveondulado, com declividade média de $8 \%$.

Amostras deformadas de solo foram coletadas em três camadas, 0 a 0,20, 0,20 a 0,40 e 0,40 a 0,60 m. Parte desse material foi utilizada para a análise granulométrica do solo e parte para a obtenção da umidade do solo no potencial de $-15.000 \mathrm{hPa}$, em câmara de Richards. Amostras não deformadas, obtidas em anéis metálicos de $0,05 \mathrm{~m}$ de diâmetro e altura, introduzidos no solo por meio de amostrador de anéis, foram utilizadas para a determinação da umidade do solo no potencial de - $60 \mathrm{hPa}$, em mesa de tensão. O material restante foi colocado para secar ao ar, sendo posteriormente peneirado em peneira com malha de 2,0 $\mathrm{mm}$, obtendo-se TFSA. Com esse material foram preenchidos, em cada camada de solo amostrado, oito cilindros de PVC, com diâmetro nominal de 0,10 e 0,25 m de altura, sob os quais se colou uma base circular plana, também de PVC. A massa de solo colocada em cada cilindro variou entre 2 e $4 \mathrm{~kg}$, conforme a densidade estabelecida no seu interior. Os valores de densidade foram obtidos de forma aleatória, não sendo fixados previamente. Procuraram-se estabelecer diferentes valores de densidade, entre 0,9 e $1,4 \mathrm{Mg} \mathrm{m}^{-3}$, variando o esforço de compactação em cada frasco, durante o preenchimento. No quadro 1 é apresentado o resumo estatístico dos valores obtidos.

Para estabelecer uma densidade diferente em cada recipiente, foi utilizada uma ferramenta que consiste de um tubo metálico com seu interior preenchido por concreto e com a base de metal de diâmetro cerca de $4 \mathrm{~mm}$ menor do que o do recipiente, com massa de aproximadamente $5 \mathrm{~kg}$. Para se estabelecer um valor de densidade, por meio da aplicação de um número de golpes, foi utilizada uma escala graduada de 0 até $0,45 \mathrm{~m}$, a qual permitiu estabelecer a altura dos golpes sobre a superfície do solo. O primeiro cilindro foi preenchido sem o processo de compactação, como forma de se obter o menor valor da densidade do solo. A partir do segundo cilindro, foram adicionadas camadas de $0,05 \mathrm{~m}$ de solo de cada vez, as quais foram compactadas com um número de golpes tanto menor quanto menos se pretendia compactar o solo e elevar o valor de Ds. Definido um valor de número de golpes e de altura do golpe para um cilindro, isso foi mantido constante em todo o seu preenchimento. Para obter a compactação máxima, o processo foi repetido por 10 vezes, com altura de queda da ferramenta de compactação de $0,45 \mathrm{~m}$, a cada camada de solo adicionada. A partir daí, os demais cilindros foram preenchidos de modo semelhante, porém variando a altura e o número de golpes, de modo a obter valores distribuídos entre o valor máximo e o mínimo, de forma aleatória.

Em seguida, os cilindros preenchidos com solo foram colocados em recipientes com água até $2 / 3$ da sua altura, para a saturação do solo, durante $24 \mathrm{~h}$. Após a saturação das amostras, introduziu-se uma sonda metálica tipo "buriable waveguide" com três hastes com 0,20 m de comprimento. Em seguida, permitiu-se a drenagem rápida da água e iniciaramse o processo de medida da massa total, utilizando uma balança de precisão, e a medida do valor de Ka, usando um equipamento de TDR (TRASE systems, inc.). Os cilindros permaneceram expostos ao ambiente no interior do laboratório e, diariamente, foram feitas entre duas ou três leituras de massa total e Ka. Ao término das leituras, determinaram-se a massa de solo seco no interior de cada recipiente, a massa do cilindro, a massa da sonda e o volume do cilindro. A partir dessas medidas, determinaram-se o valor de umidade com base em massa, o valor da densidade do solo e o valor da umidade volumétrica. O resumo estatístico desses valores encontra-se no quadro 1.

A relação entre umidade do solo e constante dielétrica foi estudada utilizando-se o modelo de regressão polinomial. O efeito da Ds foi incorporado ao modelo, por meio de procedimentos matemáticos e

Quadro 1. Resumo estatístico dos valores medidos da umidade do solo ( $\theta)$, da constante dielétrica (Ka) e da densidade do solo (Ds), nas três camadas

\begin{tabular}{|c|c|c|c|c|c|c|c|c|c|}
\hline \multirow{2}{*}{ Estatística } & \multicolumn{3}{|c|}{ Superior } & \multicolumn{3}{|c|}{ Intermediária } & \multicolumn{3}{|c|}{ Inferior } \\
\hline & $\mathbf{K a}$ & $\theta$ & Ds & $\mathbf{K a}$ & $\theta$ & Ds & $\mathbf{K a}$ & $\theta$ & Ds \\
\hline & & $\mathrm{m}^{3} \mathrm{~m}$ & $\mathrm{Mg} \mathrm{m}^{-3}$ & & & & & & \\
\hline Mínimo & 8,10 & 0,15 & 0,90 & 7,70 & 0,14 & 1,01 & 15,92 & 0,28 & 0,95 \\
\hline Máximo & 36,60 & 0,57 & 1,38 & 33,30 & 0,54 & 1,38 & 41,46 & 0,57 & 1,35 \\
\hline Média & 18,31 & 0,31 & 1,13 & 17,38 & 0,29 & 1,22 & 28,77 & 0,42 & 1,13 \\
\hline Desvio-padrão & 6,86 & 0,09 & 0,17 & 6,94 & 0,09 & 0,15 & 7,04 & 0,07 & 0,15 \\
\hline $\mathrm{CV}(\%)$ & 37,48 & 28,26 & 14,92 & 39,93 & 32,31 & 11,93 & 24,46 & 17,26 & 13,67 \\
\hline
\end{tabular}

DP: desvio-padrão; CV: coeficiente de variação (\%). 
estatísticos descritos por Silva \& Kay (1997). O modelo empregado neste trabalho foi descrito conforme a equação 1 .

$$
\begin{gathered}
\theta=\left[a_{0}+a_{1} D s\right]+\left[b_{0}+b_{1} D s\right] \\
K a+\left[c_{0}+c_{1} D s\right](K a)^{2}+\left[d_{0}+d_{1} D s\right](K a)^{3}
\end{gathered}
$$

Nessa equação, $\theta$ é a umidade do solo com base em volume $\left(\mathrm{m}^{3} \mathrm{~m}^{-3}\right)$, Ds é a densidade do solo $\left(\mathrm{Mg} \mathrm{m}^{-3}\right)$, Ka é a constante dielétrica do meio medida pelo equipamento e $a, b, c$ e $d$ são os coeficientes numéricos do modelo. Esses coeficientes empíricos foram obtidos por meio de regressão linear múltipla, utilizando-se o programa SAS (SAS, 1999). O teste t foi empregado para avaliar a significância estatística dos coeficientes estimados, conforme Netter et al. (1989).

\section{RESULTADOS E DISCUSSÃO}

O resumo estatístico dos valores de umidade do solo $(\theta$, da constante dielétrica $(\mathrm{Ka})$ e da densidade do solo (Ds), obtidos dos materiais oriundos das três camadas de solo-superior ( 0 a $0,20 \mathrm{~m}$ ), intermediária $(0,20$ a $0,40 \mathrm{~m})$ e inferior $(0,20$ a $0,40 \mathrm{~m})$ - durante o processo de secagem são apresentados no quadro 1 .

No solo estudado, o valor de $\theta$ correspondente ao potencial de $-60 \mathrm{hPa}$, adotado como o equivalente à capacidade de campo (CC), é da ordem de $0,44 \mathrm{~m}^{3} \mathrm{~m}^{-3}$, enquanto $\theta$ correspondente ao ponto de murcha permanente (PMP), ou seja, a umidade obtida para o potencial de $-15.000 \mathrm{hPa}$, é de $0,34 \mathrm{~m}^{3} \mathrm{~m}^{-3}$. Assim, a disponibilidade total de água (DTA) nesse solo é da ordem de $0,10 \mathrm{~m}^{3} \mathrm{~m}^{-3}$, o que corresponde a $1,0 \mathrm{~mm}$ de água por centímetro de profundidade de solo $\left(1,0 \mathrm{~mm} \mathrm{~cm}^{-1}=0,1 \mathrm{~m} \mathrm{~m}^{-1}\right)$. É nessa faixa de valores de umidade, ou seja, entre CC e PMP, que as medidas apresentam maior importância para os objetivos agronômicos e para o manejo da água na agricultura irrigada.

Os valores de $\theta$ e o correspondente valor de Ka obtidos a partir de todas as medidas realizadas, nas três camadas em estudo, são apresentados na figura 1. Verifica-se, que os dados mostram a dispersão semelhante entre as camadas, na faixa de valores de umidade estudada, que se mostrou mais ampla que a faixa de água disponível no solo.

A superposição dos pontos obtidos nas três camadas sugeriu não haver efeito da camada sobre o modelo. De fato, analisando os dados, constatou-se efeito estatisticamente significativo apenas do valor da densidade do solo sobre os coeficientes do modelo. Esse efeito foi considerado na geração do modelo de calibração obtido. Em razão desses resultados e aliado ao fato de que a composição granulométrica foi uniforme nas três camadas, optou-se por analisar os dados em conjunto, desconsiderando o fator camada de solo.
A relação entre Ka e $\theta$ em diferentes valores de Ds, selecionados de forma a incluir os valores extremos, é mostrada na figura 2. Com o propósito de tornar mais clara a visualização dos efeitos da variação da Ds sobre as curvas de calibração, optou-se por apresentar $\theta$ expresso com base em massa $(\theta \mathrm{g})$, como foi obtida por meio do processo de pesagem. Os valores $\theta$ correspondentes à capacidade de campo e ao ponto de murcha também estão expressos nessa base de medida, ou seja, Өg. Constata-se (Figura 2) que, com a redução do valor de Ds, a relação entre Ka e $\theta \mathrm{g}$ apresenta o aspecto característico do "S" ao qual se ajusta um modelo de terceiro grau, como foi constatado por Topp et al. (1980) e Tommazelli (1997). Nos valores mais elevados de Ds, a relação apresenta o aspecto de uma parábola com coeficiente do termo de segundo grau negativo, ou seja, concavidade voltada para baixo. Os ajustes de modelo a cada conjunto de valores obtidos com cada sonda, isoladamente, apresentaram coeficientes de determinação $\left(R^{2}\right)$ nunca inferiores a 0,95 , sendo na maior parte dos casos superiores a 0,99 . Isso revela que a técnica da TDR é extremamente sensível à variação da umidade do solo, o que expressa a exatidão associada a esse método de medida. $\mathrm{Na}$ figura 2, as linhas representam os modelos ajustados aos conjuntos de dados considerados.

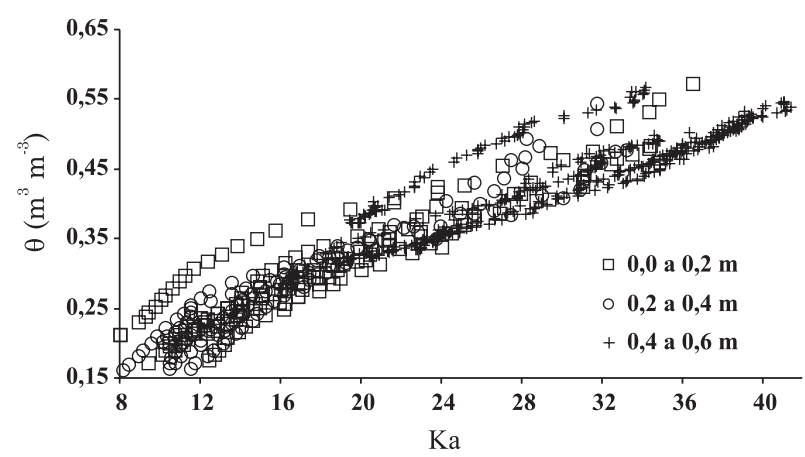

Figura 1. Valores de umidade do solo com base em volume, $\theta$, e de $\mathrm{kA}$, em diferentes valores de densidade do solo, obtidos nas três camadas de solo estudadas.

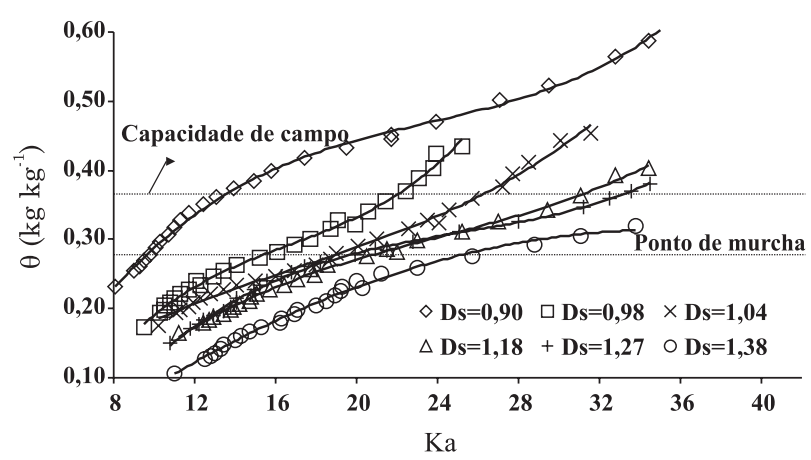

Figura 2. Valores de umidade do solo com base em massa, $\theta \mathrm{g}$, e de Ka em diferentes valores de Ds $\left(\mathrm{Mg} \mathrm{m}^{-3}\right)$, obtidos nas três camadas de solo estudadas. 
A influência da Ds nas estimativas da $\theta \mathrm{g}$ em relação aos limites de disponibilidade de água no solo pode ser avaliada na figura 2 . Em determinado valor de $\mathrm{Ka}$, os valores de $\theta \mathrm{g}$ correspondentes, obtidos nas curvas referentes aos valores extremos de Ds (0,90 e $1,38 \mathrm{Mg} \mathrm{m}^{-3}$ ), apresentam amplitude maior que a própria DTA, o que evidencia o efeito estatisticamente significativo da Ds na estimativa da umidade pela TDR. Esse efeito altamente significativo da Ds está expresso no quadro 2, na qual se mostra que os coeficientes associados à Ds no modelo apresentaram, todos, $p<0,0001$. Verifica-se na figura 2 que, para um mesmo valor de $\theta \mathrm{g}$ igual a $0,28 \mathrm{~kg} \mathrm{~kg}^{-1}$ (ponto de murcha), o valor de Ka obtido no solo com Ds de $0,9 \mathrm{Mg} \mathrm{m}^{-3}$ é de 9,8. Entretanto, para Ds de $1,38 \mathrm{Mg} \mathrm{m}^{-3}$ o valor de Ka é de 25,7. Esses resultados indicam que a variabilidade da Ds pode introduzir erro de grande magnitude quando a $\theta \mathrm{g}$ é estimada pela técnica da TDR, com o modelo matemático de ajuste da curva de calibração inserido no equipamento, o qual não leva em conta a influência da Ds.

Na figura 3 são as apresentadas curvas referentes aos valores extremos de Ds $\left(0,90\right.$ e $\left.1,38 \mathrm{Mg} \mathrm{m}^{-3}\right)$, estabelecidos neste estudo, porém expressando a umidade do solo em base de volume $(\theta)$. Pode-se constatar que as curvas se tornam mais próximas entre si, mas ainda mantêm um deslocamento relativo associado a grandes erros de medida, caso o efeito da densidade não seja incorporado ao modelo de calibração. No caso de valor de Ka igual a 14,25, por exemplo, a distância entre as curvas é equivalente à faixa de água disponível no solo. Ignorar o efeito da densidade sobre a leitura do equipamento pode levar a erro de medida equivalente a toda a faixa de água disponível nesse solo.

As duas curvas mostradas (Figura 3) permitem verificar que, na menor Ds, o modelo ajustado foi o polinomial de terceiro grau, em forma de "S". No entanto, para o maior valor de Ds o modelo torna-se uma parábola, que foi, obviamente, descrita por um modelo de segundo grau. Para esse conjunto de dados, o modelo de terceiro grau, descrito por Topp et al. (1980), é menos adequado. Essa mudança do modelo pode estar associada à drástica redução na distribuição

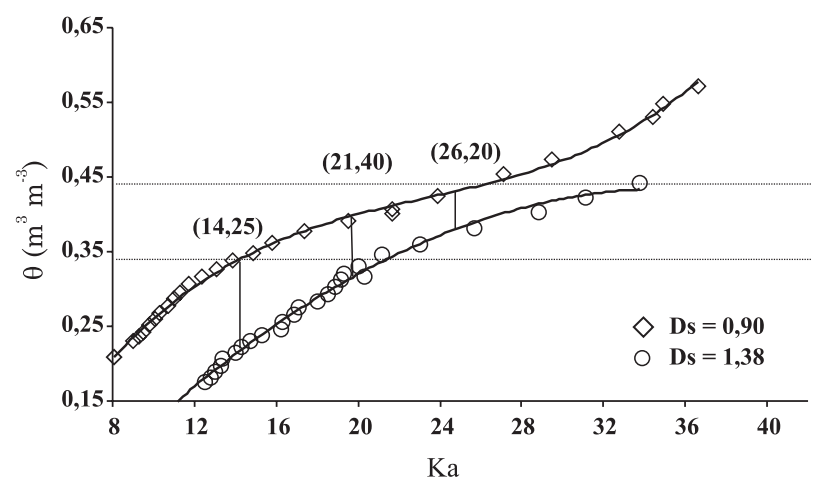

Figura 3. Valores de Ka e de $\boldsymbol{\theta}$ para valores extremos de Ds $\left(\mathrm{Mg} \mathrm{m}^{-3}\right)$, nas camadas de solo estudadas. do volume de poros, com diminuição dos maiores poros, em função do aumento da Ds. Pode-se verificar que o ponto de inflexão da curva referente à menor Ds ocorre em valores de umidade volumétrica muito próximos à capacidade de campo, a qual, por sua vez, está associada a um potencial de água no solo da ordem de $-60 \mathrm{hPa}$, que pode ser estabelecido para caracterizar a divisão entre macro e microporos (Reichardt, 1993).

Com o propósito de quantificar o efeito da Ds sobre a estimativa da umidade do solo foram tomados três valores de Ka (Figura 3) para expressar a variação de $\theta$ obtida nas curvas referentes aos valores extremos, como um percentual da DTA. Para o valor de Ka igual a 14,25 , a variação de $\theta$ corresponde a $122 \%$ da DTA. Para o valor de Ka igual a 21,40, a variação corresponde a $70 \%$ da DTA e para $\mathrm{Ka}=26,20$, a $50 \%$ da DTA. Isso mostra que, seguindo-se na direção do umedecimento do solo, as curvas se aproximam até o momento em que ocorre o ponto de inflexão, quando, então, voltam a se distanciar. Ou seja, embora a posição de uma curva em relação a outra varie conforme a umidade do solo, em toda a faixa de valores de umidade entre a saturação e a umidade inferior ao ponto de murcha, o efeito da Ds na curva de calibração é de grande magnitude, em relação ao valor da DTA.

O modelo apresentado por Topp et al. (1980) tem sido amplamente utilizado nas estimativas de $\theta$ com a TDR, independentemente de quaisquer outras variáveis. Os resultados deste trabalho indicam que a condição estrutural do solo tem influência marcante sobre o termo de terceiro grau do modelo. Como pode ser visto na figura 3 , a curva referente ao valor mínimo de Ds apresenta-se com formato característico associado ao modelo polinomial cúbico. Nesse caso, o modelo de terceiro grau mostra-se adequado. A curva referente ao valor máximo de Ds, no entanto, revela o formato de uma parábola com coeficiente do termo de segundo grau negativo. Os valores obtidos a partir do ajuste do modelo polinomial múltiplo revelaram significância apenas do termo de segundo grau, como pode ser visto no quadro 2 . Isso contesta a afirmativa de Hanke et al. (1999) de que a compactação do solo não deve alterar as medições de umidade do solo com a TDR e corrobora a hipótese apresentada por Tommaselli (1997) de que a Ds pode interferir nas estimativas de $\theta$ pelo equipamento. Em razão dessas constatações, a Ds foi incorporada ao modelo ajustado aos dados $\theta$ como função da constante dielétrica $(\mathrm{Ka})$. Foi utilizado um modelo de regressão linear múltipla, correspondente ao polinômio de segundo grau. No quadro 2 são apresentados os coeficientes do modelo, bem como o nível de significância deles e o coeficiente de determinação obtido.

O modelo ajustado evidencia o efeito de Ds nos coeficientes do modelo ajustado aos valores de $\theta$ e Ka, ou seja, comprova, estatisticamente, que Ds exerce significativa influência sobre a medida realizada com a TDR, no solo em estudo. O modelo ajustado apresentou $\mathrm{R}^{2}=0,93$ e coeficiente de variação igual a $7,2 \%$. Esse 
Quadro 2. Coeficientes do modelo de regressão linear múltipla entre umidade com base em volume $(\theta)$, constante dielétrica aparente $(\mathrm{Ka})$ e densidade do solo (Ds)

$\theta=\left[a_{0}+a_{1} \cdot d s\right]+\left[b_{0}+b_{1} \cdot d s\right] \cdot k_{a}+\left[c_{0}+c_{1} \cdot d s\right] \cdot(k a)^{2} \quad\left(R^{2}=0,93\right)$

\begin{tabular}{crcc}
\hline Coeficiente & \multicolumn{1}{c}{ Valor } & Erro-padrão & $\mathrm{p}>|\mathrm{t}|$ \\
\hline $\mathrm{a}_{\mathrm{o}}$ & 0,842538 & 0,061310 & 0,0001 \\
$\mathrm{a}_{1}$ & $-0,725175$ & 0,056294 & 0,0001 \\
$\mathrm{~b}_{0}$ & $-0,049601$ & 0,005554 & 0,0001 \\
$\mathrm{~b}_{1}$ & 0,060353 & 0,005138 & 0,0001 \\
$\mathrm{c}_{0}$ & 0,001044 & 0,000116 & 0,0001 \\
$\mathrm{c}_{1}$ & $-0,001061$ & 0,000109 & 0,0001 \\
\hline
\end{tabular}

modelo permite estimar o valor de $\theta$ a partir da leitura realizada com a TDR, desde que seja conhecida a Ds no local de medida. Na figura 4 são apresentados os valores experimentais obtidos e a superfície de valores gerados pelo modelo ajustado com a incorporação de Ds nos seus coeficientes.

Em função do significativo efeito de Ds nas estimativas de $\theta$ a partir de $\mathrm{Ka}$, uma alternativa prática para utilização da técnica da TDR nesses solos seria estabelecer intervalos de valores de Ds e estimar $\theta$ para cada intervalo de $\mathrm{Ds}$, com vistas a reduzir os erros de estimativa de $\theta$. Isso tornaria possível a utilização de modelos com capacidade para explicar melhor o fenômeno em estudo, ou seja, incrementar a exatidão nas estimativas de umidade do solo. Contudo, a aplicação em campo de situações que exigem medidas mais exatas da umidade do solo exigiria, além da leitura realizada com a TDR, informações de Ds local ou a identificação de zonas homogêneas de Ds para

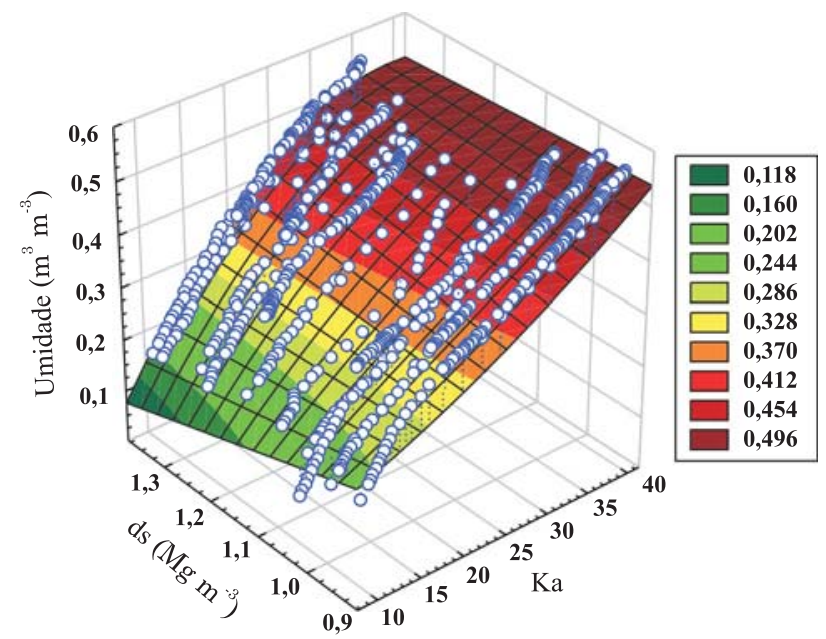

Figura 4. Valores medidos experimentalmente de $\theta$ em função de Ka em diferentes valores de Ds. A superfície apresentada corresponde ao modelo ajustado. poder efetuar a conversão da leitura de Ka em $\theta$. Ainda assim, deve-se levar em conta também o fato de que, ao se introduzir a sonda no solo, as questões referentes à instalação se tornam pertinentes, o que contribui, em muito, para elevar o nível de incerteza associado a cada leitura (Rothe et al., 1997).

A Ds exerceu influência sobre as curvas de calibração da TDR, não podendo ser ignorada para o solo em questão. Para aprimorar a exatidão da medida de umidade do solo com a TDR, o modelo da curva de calibração deve incluir a variável Ds. Caso contrário, a variação da umidade obtida com o equipamento em função da variação da Ds pode ser até maior que a disponibilidade total de água no aparelho, para determinada umidade do solo, caracterizando inadequação do emprego dessa técnica.

\section{CONCLUSÕES}

1. O efeito da Ds sobre a curva de calibração da TDR é altamente significativo.

2. Um modelo polinomial de segundo grau foi ajustado aos dados experimentais, incorporando o efeito da Ds aos seus coeficientes. Esse modelo permite estimativas adequadas da umidade do solo a partir das medidas da constante dielétrica.

3. A utilização de um modelo que não leve em conta a Ds pode levar a erros de avaliação da umidade do solo de grande magnitude, em relação ao valor da disponibilidade de água nesse solo.

\section{LITERATURA CITADA}

ALAOUI, A. \& HELBLING, A. Evaluation of soil compaction using hydrodynamic water content variation: Comparison between compacted and non-compacted soil. Geoderma, 134:97-108, 2006.

BAKER, J.M. \& ALLMARAS, R.R. System for automating and multiplexing soil moisture measurement by time domain Reflectometry. Soil Sci. Soc. Am. J., 54:1-6, 1990.

BONNELL, R.B.; BROUGHTON, R.S. \& ENRIGHT, P. The measurement of soil moisture and bulk soil salinity using time domain reflectometry. Canadian Agric. Eng., 33:225229, 1991.

BRIDGE, B.J.; SABBURG, J.; HABASH, K.O.; BALL, J.A.R. \& HANCOCK, N.H. The dielectric behaviour of clay soils and its application to time domain Reflectometry. Austr. J. Soil Res., 34:825-835, 1996.

CERNY, R. Time-domain reflectometry method and its application for measuring moisture content in porous materials: A review. Measurement, 42:329-336, 2009. 
COELHO, E.F.; VELLAME, L.M.; COELHO FILHO, M.A. \& LEDO, C.A.S. Desempenho de modelos de calibração de guias de onda acopladas a TDR e a multiplexadores em três tipos de solos. R. Bras. Ci. Solo, 30:23-30, 2006.

DAVIS, J.L. \& ANNAN, A.P. Electromagnetic detection of soil moisture. Progress Report, 1. Canadian J. Remote Sens., 3:76-86, 1977.

EMPRESA BRASILEIRA DE PESQUISA AGROPECUÁRIA EMBRAPA. Centro Nacional de Pesquisa de Solos. Sistema brasileiro de classificação de solos. Brasília, 2006. 412p.

FELLNER-FELDEGG, H. The measurement of dielectrics in the time domain. J. Phys. Chem., 73:616-623, 1969.

GARDNER, C.M.K.; DEAN, T.J. \& COOPER, J.D. Soil water content measurement with a high-frequency capacitance sensor. J. Agric. Eng. Res., 71:395-403, 1998.

HANKE, E.L.; SLOB, M.R.; OTTO, S.R.L. \& CARRILHO, P.F. Curva de retenção de água no solo - Métodos Alternativos. In: CONGRESSO BRASILEIRO DE ENGENHARIA AGRÍCOLA, SBEA, 28., Pelotas, 1999. Anais... Pelotas, 1999. CD-ROM.

HEATHMAN, G.C.; STARKS, P.J. \& BROWN, M.A. Time domain reflectometry calibration in the little Washita river experimental watershed. Soil Sci. Soc. Am. J., 67:52-61, 2003.

HIPP, J.E. Soil electromagnetic parameters as a function of frequency, soil density and soil moisture. Proc. IEEE, 62:98-103, 1974.

HOEKSTRA, P. \& DELANEY, A. Dielectric properties of soils at UHF and microwave frequencies. J. Geoph. Res., 79:1699-1708, 1974

JACKSON, S.H. In situ calibration of time domain reflectometry sensors in multiple soils. Comm. Soil Sci. Plant Anal., 35:865-878, 2004.

JACOBSEN, O.H. \& SCHJONNING, P. A laboratory calibration of time domain reflectometry for soil water measurement including effects of bulk density and texture. J. Hydrol., 151:147-157, 1993.

JONES, S.B. \& OR, D. Modeled effects on permittivity measurements of water content in high surface area porous media. Physica B, 338:284-290, 2003.

JONES, S.B.; WRAITH, J.M. \& OR, D. Time domain reflectometry measurement principles and applications. Hydrol. Proc., 16:141-153, 2002.

KAISER, D.R.; REINERT, D.J.; REICHERT, J.M. \& MINELLA, J.P.G. Dielectric constant obtainde from TDR and volumetric moisture of soils in southern Brazil. R. Bras. Ci. Solo, 34:649-658, 2010.

KAMEYAMA, K. \& MIYAMOTO, T. Measurement of solid phase permittivity for volcanic soils by time domain reflectometry. Europ. J. Soil Sci., 59:1253-1259, 2008.

KENG, J.C. \& TOPP, G.C. Measuring water content of soil columns in the laboratory: A comparison of gamma ray attenuation and TDR techniques. Canadian J. Soil Sci., 63:3-43, 1983.
MALICKI, M.A.; PLAGGE, R. \& ROTH, C.H. Improving calibration of dielectric TDR soil moisture determination taking into account the solid soil. Europ. J. Soil Sci., 47:357-366, 1996.

NETTER, J.; WASSERMAN, W. \& KUTNER, M.H. Applied linear regression models. 2.ed. Homewwod:R.D.Irwin, 1989. 245p.

NOBORIO, K. Measurement of soil water content and electrical conductivity by time domain reflectometry: A review. Comp. Electr. Agric., 31:213-237, 2001.

PETTINELLI, E.; CERETI, A.; GALLI, A. \& BELLA, F. Time domain reflectometry: Calibration techniques for accurate measurement of the dielectric properties of various materials. Rev. Sci. Instr., 73:3553-3562, 2002.

PONIZOVSKY, A.A.; CHUDINOVA, S.M. \& PACHEPSKY, Y.A. Performance of TDR calibration models as affected by soil texture. J. Hydrol., 218:35-43, 1999.

REICHARDT, K. Dinâmica da matéria e da energia em ecossistemas. Piracicaba, Escola Superior de Agricultura Luiz de Queiroz, 1993. 505p.

ROBINSON, D.A.; BELL, J.P. \& BATCHELOR, C.H. Influence of iron minerals on the determination of soil water content using dielectric techniques. J. Hydrol., 161:169-180, 1994.

ROBINSON, D.A.; JONES, S.B.; BLONQUIST, J.M. \& FRIEDMAN, S.P. A physically derived water content/ permittivity calibration model for coarse-textured, layered soils. Soil Sci. Soc. Am. J., 69:1372-1378, 2005.

ROTH, C.H.; MALICKI, M.A. \& PLAGGE, R. Empirical evaluation of the relationship between soil dielectric constant and volumetric water content as the basis for calibrating soil moisture measurements by TDR. J. Soil Sci., 43:1-13, 1992.

ROTHE, A.; WEIS, W.; KREUTZER, K.; MATTHIES, D.; HESS, U. \& ANSORGE, B. Changes in soil structure caused by the installation of time domain reflectometry probes and their influence on the measurement of soil moisture. Water Res. Res., 33:1585-1593, 1997.

SAS Institute. SAS/STAT procedure guide for personal computers. 5.ed. Cary, 1999.

SCHAAP, M.G.; LANGE, L. \& HEIMOVAARA, T.J. TDR calibration of organic forest floor media. Soil Technol., 11:205-217, 1996.

SCHNEIDER, J.M. \& FRATTA, D. Time-domain reflectometry - parametric study for the evaluation of physical properties in soils. Canadian Geotechnol. J., 46:753-767, 2009 .

SILVA, A.P. \& KAY, B.D. Estimating the least limiting water range of soils from properties and management. Soil Sci. Soc. Am. J., 61:877-883, 1997.

TIMLIN, D.J. \& PACHEPSKY, Y.A. Comparison of three methods to obtain the apparent dielectric constant from time domain reflectometry wave traces. Soil Sci. Soc. Am. J., 60:970-977, 1996. 
TODOROFF, P. \& LANGELLIER, P. Comparison of empirical and partly deterministic methods of time domain reflectometry calibration based on a study of two tropical soils. Soil Tillage Res., 45:325-340, 1998.

TOMMASELLI, J.T.G. \& BACCHI, O.O.S. Calibração de um equipamento de TDR para medida de umidade de solos. Pesq. Agropec. Bras., 36:1145-1154, 2001.

TOMMASELLI, J.T.G. Influência de algumas características do solo sobre a calibração de um aparelho de TDR (TimeDomain Reflectometry). Piracicaba, Escola Superior de Agricultura Luiz de Queiroz, 1997. 109p. (Tese de Doutorado)

TOPP, G.C.; DAVIS, J.L. \& ANNAN, A.P. Electromagnetic determination of soil water content: Measurements in coaxial transmission lines. Water Res. Res., 16:574-582, 1980.

TOPP, G.C. \& DAVIS, J.L. Measurement of soil water content using time-domain reflectometry. In: CANADIAN HYDROLOGY SYMPOSIUM, 1982, Ottawa. Proceedings... Ottawa, National Research Council of Canada, 1982. p.269-287.

TOPP, G.C. \& REYNOLDS, W.D. Time domain reflectometry: a seminal technique for measuring mass and energy in soil. Soil Tillage Res., 47:125-132, 1998.
TOPP, G.C.; DAVIS, J.L.; BAILEY, W.G. \& ZEBCHUK, W.D. The measurement of soil water content using a portable TDR hand probe. Ottawa. Canadian J. Soil Sci., 64:313321,1984

TSEGAYE, T.D.; TEDESSE, W.; COLEMAN, T.L.; JACKSON, T.J. \& TEWOLDE, H. Calibration and modification of impedance probe for near surface soil moisture measurements. Canadian J. Soil Sci., 84:237-243, 2004.

WALKER, J.P.; WILLGOOSE, G.R. \& KALMA, J.D. In situ measurement of soil moisture: A comparison of techniques. J. Hydrol., 293:85-99, 2004.

WANG, D.; YATES, S.R. \& ERNST, F.F. Determining soil hydraulic properties using tension infiltrometers, time domain reflectometry, and tensiometers. Soil Sci. Soc. Am. J., 62:318-325, 1998.

WEITZ, A.M.; GRAUEL, W.T.; KELLER, M. \& VELDKAMP, E. Calibration fo time domain reflectometry technique using undisturbed soilsamples from humid tropical soils of volcanic origin. Water Res. Res., 33:1241-1249, 1997.

ZEGELIN, S.J.; WHITE, J. \& JENKINS, D.R. Improved fiel probes for soil water content and electrical conductivity measurement using time domain reflectometry. Water Res. Res., 25:2367-2376, 1989. 
